\title{
FINELY HARMONIC FUNCTIONS \\ WITH FINITE DIRICHLET INTEGRAL WITH RESPECT TO THE GREEN MEASURE
}

BY

BERNT ØKSENDAL

ABSTRaCT. We consider finely harmonic functions $h$ on a fine, Greenian domain $V \subset \mathbf{R}^{d}$ with finite Dirichlet integral wrt $G m$, i.e.

$$
\int_{V}|\nabla h(y)|^{2} G(x, y) d m(y)<\infty \quad \text { for } x \in V,
$$

where $m$ denotes the Lebesgue measure, $G(x, y)$ the Green function. We use Brownian motion and stochastic calculus to prove that such functions $h$ always have boundary values $h^{*}$ along a.a. Brownian paths. This partially extends results by Doob, Brelot and Godefroid, who considered ordinary harmonic functions with finite Dirichlet integral wrt $m$ and Green lines instead of Brownian paths.

As a consequence of Theorem 1 we obtain several properties equivalent to (*), one of these being that $h$ is the harmonic extension to $V$ of a random "boundary" function $h^{*}$ (of a certain type), i.e. $h(x)=E^{x}\left[h^{*}\right]$ for all $x \in V$. Another application is that the polar sets are removable singularity sets for finely harmonic functions satisfying (*). This is in contrast with the situation for finely harmonic functions with finite Dirichlet integral wrt $m$.

1. Introduction and statement of results. Properties of harmonic functions with finite Dirichlet integral have been studied by several authors. In 1962, Doob [5], extending earlier works by Brelot and Godefroid, proved that a harmonic function $h$ on a domain $V$ in $\mathbf{R}^{d}(d \geqslant 2)$ admitting a Green function and with a finite Dirichlet integral, i.e.

$$
\int_{V}|\nabla h|^{2} d m<\infty
$$

(where $m$ denotes Lebesgue measure in $\mathbf{R}^{d}$ ) always has a fine boundary function $h^{*}$ and $h \rightarrow h^{*}$ along the Green lines of $V$. Doob (and Brelot and Godefroid) used a measure on the space of all Green lines.

In this article we use Brownian motion and stochastic calculus to prove a result of this type and establish a corresponding $L^{2}$-isometry (Theorem 1 ) in the more general situation when $h$ is a finely harmonic function on a fine domain $V$ in $\mathbf{R}^{d}$ with a Green function $G$. The assumption that $h$ has a finite Dirichlet integral is replaced by the assumption that

$$
\int_{V}|\nabla h(y)|^{2} G(x, y) d m(y)<\infty \quad \text { for all } x \in V,
$$

Received by the editors April 16, 1984.

1980 Mathematics Subject Classification. Primary $60 \mathrm{~J} 45$. 
i.e. that $h$ has a finite Dirichlet integral wrt the Green measure. (It is known (Debiard and Gaveau [4]) that $\nabla h$ exists a.e. wrt $m$ on $V$.) In the case when $h$ is harmonic in the ordinary sense on an ordinary Greenian domain $V$ then (1.1) is a stronger assumption than (1.2), because $G(x, y) \rightarrow 0$ as $y \rightarrow \partial V$ (the boundary of $V)$ and the singularity of $G(x, y)$ at $y=x$ is $m$-integrable.

For example, if $V$ is the unit disc $D \subset \mathbf{R}^{2}$ then a harmonic function $h$ on $D$ satisfies (1.1) if and only if the Fourier coefficients $\hat{h}^{*}(n)$ of its boundary function $h^{*}$ satisfy

$$
\sum_{-\infty}^{\infty}\left|\hat{h}^{*}(n)\right|^{2}|n|<\infty,
$$

while $h$ satisfies (1.2) if and only if

$$
\sum_{-\infty}^{\infty}\left|\hat{h}^{*}(n)\right|^{2}<\infty
$$

((1.3) can be found, for example, in M. Fukushima [12, p. 12], while (1.4) is a consequence of Theorem 2). In the general fine situation it turns out that (1.1) implies that (1.2) holds quasi-everywhere, i.e. everywhere outside some polar set. To see this let $W$ be a bounded subset of $V$ and assume that (1.1) holds. Then by the Fubini theorem

$$
\begin{aligned}
\int_{W}\left(\int_{V}|\nabla h(y)|^{2} G(x, y) d m(y)\right) d m(x) \\
=\int_{V}|\nabla h(y)|^{2}\left(\int_{W} G(x, y) d m(x)\right) d m(y)<\infty,
\end{aligned}
$$

since $\sup _{y}\left(\int_{W} G(x, y) d m(x)\right)<\infty$. So (1.2) holds for a.a. $x \in W$ wrt $m$. In particular, the function $H(x)=\int_{V}|\nabla h(y)|^{2} G(x, y) d m(y)$ is not infinite everywhere in $V$. But then it follows from Theorem 2.4 in Fuglede [11] that $H(x)$ is a fine potential in $V$ and therefore finite quasi-everywhere, as asserted.

As a consequence of Theorem 1 we obtain several properties equivalent to (1.2), one of these being that $h$ is the harmonic extension to $V$ of a random function $h^{*}$ (of a certain type), i.e. $h(x)=E^{x}\left[h^{*}\right]$ for all $x \in V$ (Theorem 2). Another application is that the polar sets are removable singularity sets for a finely harmonic function $h$ satisfying (1.2) (Theorem 3). This result is in contrast with the situation for finely harmonic functions $h$ satisfying (1.1). In this case it is known that polar sets need not be removable singularity sets (see Fuglede [10, Théorème 12 and p. 153]). Thus the condition (1.1) does not imply (1.2) in general.

2. Boundary behaviour. In the following, $B_{t}(\omega), \omega \in \Omega, t \geqslant 0$, will denote Brownian motion in $\mathbf{R}^{d}(d \geqslant 2)$. The probability law of $B_{t}$ starting at $x \in \mathbf{R}^{d}$ is denoted by $P^{x}$, and $E^{x}$ is the expectation operator wrt $P^{x}$.

For a finely open set $V \subset \mathbf{R}^{d}$ we will let $\tau_{V}=\inf \left\{t>0 ; B_{t} \in V\right\}$ be the first exit time from $V\left(\tau_{V}=\infty\right.$ if $B_{t} \in V$ for all $\left.t>0\right)$. If $\tau_{V}<\infty$ a.s. the harmonic measure $\lambda_{x}^{V}$ at $x$ wrt $V$ is defined by

$$
\int_{\partial V} f d \lambda_{x}^{V}=E^{x}\left[f\left(B_{\tau_{V}}\right)\right]
$$

if $f$ is a bounded, continuous real function on $\partial V$. 
The Green function $G(x, y)$ of fine domain $V \subset \mathbf{R}^{d}$ is defined by

$$
G(x, y) d m(y)=\int_{0}^{\infty} P^{x}\left[B_{s} \in d y, s<\tau_{V}\right] d s,
$$

provided the integral converges. Intuitively, $G(x, y) d m(y)$ is the expected length of time Brownian motion starting at $x$ stays in $d m(y)$ before it exits from $V$. See Chung [3] for more information. The domain $V$ is called Greenian if $\int_{K} G(x, y) d m(y)<\infty$ for all compact sets $K$ and all $x \in V$. All finely open sets in $\mathbf{R}^{d}$ for $d \geqslant 3$ are Greenian. If $V \subset \mathbf{R}^{2}$ then $V$ is Greenian if and only if $V$ has a nonpolar complement.

LEMMA 1. Let $h$ be a finely harmonic function in a finely open set $V \subset \mathbf{R}^{d}$ with $a$ Green function $G$. Let $\tau_{V}$ be the first exit time from $V$. Then

$$
E^{x}\left[\int_{0}^{\tau_{V}}\left|\nabla h\left(B_{s}\right)\right|^{2} d s\right]=\int_{V}|\nabla h(y)|^{2} G(x, y) d m(y) \quad \text { for all } x \in V .
$$

Proof. By the Fubini theorem we have ( $\chi$ denotes the indicator function)

$$
\begin{aligned}
E^{x}\left[\int_{0}^{\tau_{V}}\left|\nabla h\left(B_{s}\right)\right|^{2} d s\right] & =E^{x}\left[\int_{0}^{\infty}\left|\nabla h\left(B_{s}\right)\right|^{2} \chi_{\left[0, \tau_{V}\right)}(s) d s\right] \\
& =\int_{0}^{\infty}\left(\int_{V}|\nabla h(y)|^{2} \cdot P^{x}\left[B_{s} \in d y, s<\tau_{V}\right]\right) d s \\
& =\int_{V}|\nabla h(y)|^{2}\left(\int_{0}^{\infty} P^{x}\left[B_{s} \in d y, s<\tau_{V}\right] d s\right) \\
& =\int_{V}|\nabla h(y)|^{2} G(x, y) d m(y) .
\end{aligned}
$$

which proves Lemma 1.

LEMMA 2. Let $f$ be a real, finely continuous function on a finely open set $V \subset \mathbf{R}^{d}$. Then for all $x \in V$ the function

$$
t \rightarrow f\left(B_{t}(\omega)\right)
$$

is continuous on $\left[0, \tau_{V}\right)$, for a.a. $\omega \in \Omega$ wrt $P^{x}$.

Proof. Since $f$ is finely continuous, it is quasi-continuous, i.e. for all $\eta>0$ there exists an open set $G \subset \mathbf{R}^{d}$ with $C(G)<\eta$ and $f$ is continuous (in the ordinary topology) outside $G$, where $C$ denotes the capacity associated to the kernel $k(x)=$ $|x|^{2-d}$ if $d \geqslant 3$ and $k(x)=-\log |x|$ if $d=2$. (See Brelot [1, Theorem IV, 3, p. 25].) So if $U \subset V$ is a Greenian domain in $\mathbf{R}^{d}$ and $x \in U, \varepsilon>0$, we can find an open set $G$ such that $P^{x}\left\{\exists t, 0<t<\tau_{U}, X_{t} \in G\right\}<\varepsilon$ and $f \mid \mathbf{R}^{d} \backslash G$ is continuous. In particular,

$$
P^{x}\left\{t \rightarrow f\left(X_{t}\right) \text { is continuous, } t<\tau_{U}\right\}>1-\varepsilon .
$$

Since $U$ and $\varepsilon$ was arbitrary we obtain that $t \rightarrow f\left(X_{t}\right)$ is continuous a.s. $P^{x}$ on $\left\{t<\tau_{V}\right\}$.

LEMMA 3. Let $U \subset \mathbf{R}^{d}$ be finely open and let $\tau$ be a stopping time. Then for a.e. $\omega$ wrt $P^{x}$ we have:

If $B_{\tau}(\omega) \in U$ then there exists $\varepsilon>0$ such that $B_{t}(\omega) \in U$ for all $t \in(\tau(\omega)-\varepsilon, \tau(\omega))$. 
PROof. Since the fine topology is completely regular we can for each $x \in U$ find a finely continuous function $y \rightarrow f_{x}(y)$ on $\mathbf{R}^{d}$ such that $0 \leqslant f_{x} \leqslant 1, f_{x} \equiv 1$ on $\mathbf{R}^{d} \backslash U$ and $f_{x}(x)=0$. Let $D_{x} \subset U$ be a fine neighbourhood of $x$ such that $f_{x}<\frac{1}{2}$ on $D_{x}$. The family $\left\{D_{x}\right\}_{x \in U}$ covers $U$, so by Doob's quasi-Lindelöf principle [5] we can find a countable subfamily $\left\{D_{x_{k}}\right\}_{k=1}^{\infty}$ such that

$$
K=U \backslash \bigcup_{k=1}^{\infty} D_{x_{k}}
$$

is polar. Put

$$
f=\sum_{k=1}^{\infty} 2^{-k} f_{x_{k}}
$$

Then $f$ is finely continuous, $f \equiv 1$ on $\mathbf{R}^{d} \backslash U$, and $f<1$ on $U \backslash K$. Assume $B_{\tau} \in U$. Since $K$ is polar, $B_{\tau} \in K$, and therefore $f\left(B_{\tau}\right)<1$, a.s. By Lemma $2, t \rightarrow f\left(B_{t}\right)$ is continuous a.s. So for a.a. $\omega$ there exists $\varepsilon>0$ such that $f\left(B_{t}\right)<1$ for $\tau-\varepsilon<t<\tau$. This implies that $B_{t} \in U$ for $\tau-\varepsilon<t<\tau$ and Lemma 3 is proved.

LEMMA 4. Let $h$ be a finely harmonic function in a fine domain $V \subset \mathbf{R}^{d}$. Choose $x \in V$. Then we can find an increasing sequence of fine bounded domains $V_{n}$ with $\bar{V}_{n} \subset V$ such that, with $\tau_{n}=\tau_{V_{n}}$, we have

$$
\begin{gathered}
\tau_{n} \uparrow \tau_{V} \text { a.s. } P^{x} \quad \text { as } n \rightarrow \infty, \\
E^{x}\left[h^{2}\left(B_{t \wedge \tau_{n}}\right)\right]=h^{2}(x)+E^{x}\left[\int_{0}^{t \wedge \tau_{n}}\left|\nabla h\left(B_{s}\right)\right|^{2} d s\right]<\infty
\end{gathered}
$$

for all $n$,

$$
h \text { is continuous on } V_{n} \text {, }
$$

and

$$
h\left(B_{t \wedge \tau_{n}}\right)=h(x)+\int_{0}^{t \wedge \tau_{n}} \nabla h\left(B_{s}\right) d B_{s} \text { a.s. } P^{x}
$$

Proof. Choose $z \in V$. Then there exists a fine bounded neighbourhood $U_{z} \ni z$ with compact closure $\bar{U}_{z} \subset V$ and a sequence of functions $h_{m}$ harmonic (in the ordinary sense) in a neighbourhood of $\bar{U}_{z}$ such that $h_{m} \rightarrow h$ uniformly on $\bar{U}_{z}$ (Fuglede [8, Theorem 4.1]). Put $\tau=\tau_{U_{z}}$. Then by Ito's formula

$$
h_{m}\left(B_{t \wedge \tau}\right)-h_{m}(z)=\int_{0}^{t \wedge \tau} \nabla h_{m}\left(B_{s}\right) d B_{s} \text { for all } n, t \text {, a.s. } P^{z} \text {. }
$$

So by the basic isometry for Ito integrals

$$
E^{z}\left[\left(h_{m}\left(B_{t \wedge \tau}\right)-h_{m}(z)\right)^{2}\right]=E^{z}\left[\int_{0}^{t \wedge \tau}\left|\nabla h_{m}\left(B_{s}\right)\right|^{2} d s\right],
$$

i.e.

$$
E^{z}\left[h_{m}^{2}\left(B_{t \wedge \tau}\right)\right]=h_{m}^{2}(z)+E^{z}\left[\int_{0}^{t \wedge \tau}\left|\nabla h_{m}\left(B_{s}\right)\right|^{2} d s\right],
$$

since

$$
E^{z}\left[h_{m}\left(B_{t \wedge \tau}\right)\right]=h_{m}(z) \text { for all } m \text {. }
$$


Letting $m \rightarrow \infty$ we obtain, using Lemma 1 and Théorème 2 in [4],

$$
E^{z}\left[h^{2}\left(B_{t \wedge \tau}\right)\right]=h^{2}(z)+E^{z}\left[\int_{0}^{t \wedge \tau}\left|\nabla h\left(B_{s}\right)\right|^{2} d s\right]<\infty
$$

and

$$
h\left(B_{t \wedge \tau}\right)=h(z)+\int_{0}^{t \wedge \tau} \nabla h\left(B_{s}\right) d B_{s} \text { a.s. } P^{z} \text {. }
$$

The family $\left\{U_{z}\right\}_{z \in V}$ covers $V$, so by Doob's quasi-Lindelöf principle [5] we can find a countable subfamily denoted by $\left\{W_{n}\right\}$ such that $W_{1}=U_{x}$ and

$$
\bigcup_{n=1}^{\infty} W_{n}=V \backslash K
$$

where $K$ is a polar set. Now define

$$
V_{n}=\bigcup_{k=1}^{n} W_{k}, \quad n=1,2, \ldots
$$

Since $K$ is polar, (2.3) holds. We prove (2.4) and (2.5) by induction: The argument above proves that $(2.4)$ and $(2.5)$ holds for $n=1$. To prove the induction step assume that it holds for $n=k$. Put $S_{0}=\tau_{k}, T=\tau_{k+1}\left(=\tau_{V_{k} \cup W_{k+1}}\right)$. Define

$$
\begin{aligned}
& S_{1}=\inf \left\{t>S_{0} ; B_{t} \notin W_{k+1}\right\}, \\
& S_{2}=\inf \left\{t>S_{1} ; B_{t} \notin V_{k}\right\},
\end{aligned}
$$

and, inductively,

$$
\begin{aligned}
& S_{2 j+1}=\inf \left\{t>S_{2 j} ; B_{t} \notin W_{k+1}\right\}, \\
& S_{2 j+2}=\inf \left\{t>S_{2 j+1} ; B_{t} \notin V_{k}\right\}, \quad j=0,1,2, \ldots
\end{aligned}
$$

Then $\left\{S_{j}\right\}$ is an increasing sequence of stopping times. Since $S_{j} \leqslant T<\infty$ a.s. the limit

$$
S=\lim _{j \rightarrow \infty} S_{j}
$$

exists a.s. and $S \leqslant T$. Since $B_{S_{2 j+1}} \in \partial_{f} W_{k+1}$ for all $j$ ( $\partial_{f}$ denotes fine boundary) we must have $B_{s} \notin W_{k+1}$ a.s., by Lemma 3. Similarly $B_{s} \notin V_{k}$ a.s. Thus $S \geqslant T$ and therefore $S=T$. Therefore it suffices to prove that

$$
E^{x}\left[h^{2}\left(B_{t \wedge s_{j}}\right)\right]=h^{2}(x)+E^{x}\left[\int_{0}^{t \wedge s_{j}}\left|\nabla h\left(B_{s}\right)\right|^{2} d s\right]<\infty \quad \text { for all } j
$$

and

$$
h\left(B_{t \wedge S_{j}}\right)=h(x)+\int_{0}^{t \wedge S_{j}} \nabla h\left(B_{s}\right) d B_{s} \text { a.s. } P^{x}, \text { for all } j .
$$

For if (2.6) and (2.7) are established then the induction steps of (2.4) and (2.5) follows by bounded convergence if we let $j \rightarrow \infty$. (Recall that $h$ is bounded on $V_{k+1}$.)

We establish (2.6) and (2.7) by induction on $j$. The strong Markov property states that if $\tau$ is a stopping time and $\eta$ is measurable wrt $\left\{B_{s} ; s \geqslant 0\right\}$, then

$$
E^{x}\left[\theta_{\tau} \eta \mid B_{\tau}\right]=E^{B_{\tau}}[\eta] \text {, }
$$


where $\theta_{\tau}$ is the shift operator:

$$
\theta_{t}\left(g_{1}\left(B_{t_{1}}\right) \cdots g_{i}\left(B_{t_{i}}\right)\right)=g_{1}\left(B_{t_{1}+t}\right) \cdots g_{i}\left(B_{t_{i}+t}\right) \text {. }
$$

(See Dynkin [7, Theorem 3.11, p. 100], or Øksendal [13, (7.15)].) Assume (2.6) holds for a given $j$. For simplicity put $a=t \wedge S_{j}, b=t \wedge S_{j+1}$. Then, using (7.16) in [13]

$$
\begin{aligned}
E^{x}\left[h^{2}\left(B_{b}\right)\right] & =E^{x}\left[E^{x}\left[h^{2}\left(B_{b}\right) \mid B_{a}\right]\right]=E^{x}\left[E^{B_{a}}\left[h^{2}\left(B_{b}\right)\right]\right] \\
& =E^{x}\left[h^{2}\left(B_{a}\right)+E^{B_{a}}\left[\int_{0}^{b}\left|\nabla h\left(B_{s}\right)\right|^{2} d s\right]\right] \\
& =h^{2}(x)+E^{x}\left[\int_{0}^{a}\left|\nabla h\left(B_{s}\right)\right|^{2} d s\right]+E^{x}\left[E^{B_{a}}[\psi]\right],
\end{aligned}
$$

where $\psi=\int_{0}^{b}\left|\nabla h\left(B_{s}\right)\right|^{2} d s=\int_{0}^{\infty}\left|\nabla h\left(B_{s}\right)\right|^{2} \chi_{[s, \infty)}(b) d s$. Since

$$
E^{x}\left[E^{B_{a}}[\psi]\right]=E^{x}\left[E^{x}\left[\theta_{a} \psi \mid B_{a}\right]\right]=E^{x}\left[\theta_{a} \psi\right]
$$

and

$$
\begin{aligned}
\theta_{a} \psi & =\int_{0}^{\infty}\left|\nabla h\left(B_{a+s}\right)\right|^{2} \cdot \chi_{[a+s, \infty)}(b) d s \\
& =\int_{a}^{\infty}\left|\nabla h\left(B_{u}\right)\right|^{2} \chi_{[u, \infty)}(b) d u=\int_{a}^{b}\left|\nabla h\left(B_{s}\right)\right|^{2} d s,
\end{aligned}
$$

we obtain from (2.8) that

$$
E^{x}\left[h^{2}\left(B_{b}\right)\right]=h^{2}(x)+E^{x}\left[\int_{0}^{b}\left|\nabla h\left(B_{s}\right)\right|^{2} d s\right]
$$

which establishes the induction step of (2.6). Observe that by Lemma 1

$$
\begin{aligned}
E^{x}\left[\int_{0}^{\tau_{n}}\left|\nabla h\left(B_{s}\right)\right|^{2} d s\right] & =\int_{V_{n}}|\nabla t|^{2} G(x, y) d m(y) \\
& \leqslant \sum_{i=1}^{n} \int_{W_{i}}|\nabla h|^{2} G(x, y) d m(y)<\infty
\end{aligned}
$$

Next assume that (2.7) holds for a given $j$. Then

$$
\begin{aligned}
& E^{x}\left[\left(h\left(B_{b}\right)-h(x)-\int_{0}^{b} \nabla h\left(B_{s}\right) d B_{s}\right)^{2}\right] \\
& \quad=2 E^{x}\left[\left(\int_{0}^{b} \nabla h\left(B_{s}\right) d B_{s}\right)^{2}\right]-2 E^{x}\left[h\left(B_{b}\right) \cdot \int_{0}^{b} \nabla h\left(B_{s}\right) d B_{s}\right],
\end{aligned}
$$


where

$$
\begin{aligned}
& E^{x}\left[h\left(B_{b}\right) \cdot \int_{0}^{b} \nabla h\left(B_{s}\right) d B_{s}\right]=E^{x}\left[E^{x}\left[h\left(B_{b}\right)\left(\int_{0}^{a}+\int_{a}^{b}\right) \mid B_{a}\right]\right] \\
& =E^{x}\left[\left(\int_{0}^{a} \nabla h\left(B_{s}\right) d B_{s}\right) \cdot E^{B_{a}}\left[h\left(B_{b}\right)\right]\right]+E^{x}\left[E^{B_{a}}\left[B_{b} \cdot \int_{0}^{b} \nabla h\left(B_{s}\right) d B_{s}\right]\right] \\
& =E^{x}\left[\left(\int_{0}^{a} \nabla h\left(B_{s}\right) d B_{s}\right) h\left(B_{a}\right)\right]+E^{x}\left[E^{B_{a}}\left[\left(\int_{0}^{b} \nabla h\left(B_{s}\right) d B_{s}\right)^{2}\right]\right] \\
& =E^{x}\left[\left(\int_{0}^{a} \nabla h\left(B_{s}\right) d B_{s}\right)^{2}\right]+E^{x}\left[E^{x}\left[\left(\int_{a}^{b} \nabla h\left(B_{s}\right) d B_{s}\right)^{2} \mid B_{a}\right]\right] \\
& =E^{x}\left[\int_{0}^{a}\left|\nabla h\left(B_{s}\right)\right|^{2} d s\right]+E^{x}\left[\int_{a}^{b}\left|\nabla h\left(B_{s}\right)\right|^{2} d B_{s}\right] \\
& =E^{x}\left[\int_{0}^{b}\left|\nabla h\left(B_{s}\right)\right|^{2} d s\right]
\end{aligned}
$$

and the induction step of (2.7) follows.

That completes the proof of Lemma 4.

REMARK. Note that the proof gives that (2.3)-(2.5) still hold if we replace $x$ by any point $y \in \cup_{n=1}^{\infty} V_{n}$ or if we add to the family $\left\{W_{n}\right\}$ any finite collection of the sets $U_{z}$.

To each $x \in V$ we associate a sequence of finely open sets $V_{n}=V_{n}^{x}$ and stopping times $\tau_{n}=\tau_{n}^{x}$ as in Lemma 4 . Let $\mathfrak{B}_{n}^{(x)}$ denote the $\sigma$-algebra of subsets of $\Omega$ generated by the random variables $\left\{B_{\tau_{k}^{x}} ; k \geqslant n\right\}$, completed wrt the measure $P^{x}$. Define

$$
\mathfrak{B}^{(x)}=\bigcap_{\mathfrak{B}_{n}^{(x)}},
$$

i.e. $\mathfrak{B}^{x}$ is the tail field of the sequence $\left\{B_{\tau_{n}^{x}}\right\}$. Finally, put

$$
\mathfrak{B}=\mathfrak{B}^{V}=\bigcap_{x \in V} \mathfrak{B}^{(x)} .
$$

We are now ready to prove the main result about stochastic boundary values of finely harmonic functions:

THEOREM 1. Let $h$ be a finely harmonic function in a fine domain $V \subset \mathbf{R}^{d}$ with $a$ Green function $G$, and assume that

$$
\int_{V}|\nabla h(y)|^{2} G(x, y) d m(y)<\infty \quad \text { for all } x \in V .
$$

Then there exists a function $h^{*} \in L^{2}\left(P^{x}\right)$ for all $x$ such that

$$
\lim _{t \uparrow \tau_{\nu}} h\left(B_{t}\right)=h^{*} \text { a.s. } P^{x}
$$

and

$$
E^{x}\left[\left(h\left(B_{t \wedge \tau_{\nu}}\right)-h^{*}\right)^{2}\right] \rightarrow 0 \text { as } t \uparrow \infty, \text { for all } x \in V .
$$

We may regard $h^{*}$ as a generalized (random) boundary value function of $h$, in the sense that $h^{*}$ is measurable wrt the tail field $\mathfrak{B}^{V}$ and $h$ is the "harmonic extension" of $h^{*}$ to 
V, i.e.

$$
h(x)=E^{x}\left[h^{*}\right] \text { for all } x \in V .
$$

Moreover, for all $x \in V$ we have

$$
h^{*}(\omega)=h(x)+\int_{0}^{\tau_{V}} \nabla h\left(B_{s}\right) d B_{s} \text { a.s. } P^{x}
$$

and

$$
E^{x}\left[\left(h^{*}\right)^{2}\right]=h^{2}(x)+\int_{V}|\nabla h(y)|^{2} G(x, y) d m(y) .
$$

Proof. Fix $x \in V$. Choose finely open sets $V_{n}=V_{n}^{x}$ with exit times $\tau_{n}=\tau_{n}^{x}$ as in Lemma 4. Let $n>m$.

Then

$$
\begin{aligned}
E^{x}\left[h\left(B_{\tau_{n}}\right) h\left(B_{\tau_{m}}\right)\right] & =E^{x}\left[E^{x}\left[h\left(B_{\tau_{n}}\right) h\left(B_{\tau_{m}}\right) \mid B_{\tau_{m}}\right]\right] \\
& =E^{x}\left[h\left(B_{\tau_{m}}\right) E^{x}\left[h\left(B_{\tau_{n}}\right) \mid B_{\tau_{m}}\right]\right] \\
& =E^{x}\left[h^{2}\left(B_{\tau_{m}}\right)\right] .
\end{aligned}
$$

Therefore

$$
\begin{aligned}
E^{x}\left[\left(h\left(B_{\tau_{n}}\right)-h\left(B_{\tau_{m}}\right)\right)^{2}\right] & =E^{x}\left[h^{2}\left(B_{\tau_{n}}\right)\right]-2 E^{x}\left[h\left(B_{\tau_{n}}\right) h\left(B_{\tau_{m}}\right)\right]+E^{x}\left[h^{2}\left(B_{t_{m}}\right)\right] \\
& =E^{x}\left[h^{2}\left(B_{\tau_{n}}\right)\right]-E^{x}\left[h^{2}\left(B_{\tau_{m}}\right)\right]=E^{x}\left[\int_{\tau_{m}}^{\tau_{n}}\left|\nabla h\left(B_{s}\right)\right|^{2} d s\right] \\
& \leqslant \int_{V_{n} \backslash V_{m}}|\nabla h(y)|^{2} G(x, y) d m(y) \rightarrow 0 \quad \text { as } m, n \rightarrow \infty .
\end{aligned}
$$

So the sequence of functions $h_{n}=h\left(B_{\tau_{n}}\right)$ converges in $L^{2}\left(P^{x}\right)$ to a function $h^{x} \in L^{2}\left(P^{x}\right)$. In particular

$$
h(x)=\lim _{n \rightarrow \infty} E^{x}\left[h\left(B_{\tau_{n}}\right)\right]=E^{x}\left[h^{x}\right] .
$$

By Lemma 4 we have

$$
h_{n}=h(x)+\int_{0}^{\tau_{n}} \nabla h_{n}\left(B_{s}\right) d B_{s} \text { a.s. } P^{x}
$$

and

$$
\begin{aligned}
E^{x}\left[h_{n}^{2}\right] & =h^{2}(x)+E^{x}\left[\int_{0}^{\tau_{n}}\left|\nabla h\left(B_{s}\right)\right|^{2} d s\right] \leqslant h^{2}(x)+E^{x}\left[\int_{0}^{\tau}\left|\nabla h\left(B_{s}\right)\right|^{2} d s\right] \\
& =h^{2}(x)+\int_{V}|\nabla h|^{2}(y) G(x, y) d m(y)<\infty \text { for all } n .
\end{aligned}
$$

The family $\left\{h_{n}\right\}$ constitutes a martingale wrt the $\sigma$-algebras $\mathscr{F}_{n}$ generated by $B_{t}$, $t \leqslant \tau_{n}$. So by the martingale convergence theorem $P^{x}\left(\lim _{n \rightarrow \infty} h_{n}\right.$ exists $)=1$ and we conclude that

$$
h^{x}(\omega)=\lim _{n \rightarrow \infty} h_{n}(\omega) \text { a.s. } P^{x}
$$


Moreover, since $\tau_{n} \uparrow \tau_{V}$ a.s. $P^{x}$ we obtain, from (2.15) and (2.16), by letting $n \rightarrow \infty$

$$
h^{x}(\omega)=h(x)+\int_{0}^{\tau_{V}}(\nabla h)\left(B_{s}\right) d s
$$

and

$$
E^{x}\left[\left(h^{x}\right)^{2}\right]=h^{2}(x)+\int_{V}|\nabla h(y)|^{2} G(x, y) d m(y) .
$$

Note that (2.14), (2.18) and (2.19) are identical to (2.11), (2.12) and (2.13) except that $h^{*}$ is replaced by $h^{x}$. Next we establish (2.9) and (2.10) with $h^{*}$ replaced by $h^{x}$ : For all $t \geqslant 0$ and $n=1,2, \ldots$ we get by Lemma 4

$$
h\left(B_{t \wedge \tau_{n}}\right)=h(x)+\int_{0}^{t \wedge \tau_{n}}(\nabla h)\left(B_{s}\right) d B_{s} \text { a.s. } P^{x}
$$

and

$$
E^{x}\left[h^{2}\left(B_{t \wedge \tau_{n}}\right)\right]=h^{2}(x)+E^{x}\left[\int_{0}^{t \wedge \tau_{n}}\left|\nabla h\left(B_{s}\right)\right|^{2} d s\right] .
$$

The same procedure as above gives, for $n>m$,

$$
E^{x}\left[\left(h\left(B_{t \wedge \tau_{n}}\right)-h\left(B_{t \wedge \tau_{m}}\right)\right)^{2}\right]=E^{x}\left[\int_{t \wedge \tau_{m}}^{t \wedge \tau_{n}}\left|\nabla h\left(B_{s}\right)\right|^{2} d s\right] \rightarrow 0 \quad \text { as } m, n \rightarrow \infty .
$$

So letting $n \rightarrow \infty$ in (2.20), (2.21) and using (2.17), we conclude

$$
h\left(B_{t \wedge \tau_{V}}\right)=h(x)+\int_{0}^{t \wedge \tau_{V}}(\nabla h)\left(B_{s}\right) d B_{s} \text { a.s. } P^{x}
$$

and

$$
E^{x}\left[h^{2}\left(B_{t \wedge \tau_{V}}\right)\right]=h^{2}(x)+E^{x}\left[\int_{0}^{t \wedge \tau_{V}}\left|\nabla h\left(B_{s}\right)\right|^{2} d s\right]
$$

where $h\left(B_{t \wedge \tau_{V}}\right)$ is interpreted as $h^{x}$ if $t=\tau_{V}$. From (2.22) we get, for $t>s$

$$
E^{x}\left[\left(h\left(B_{t \wedge \tau_{V}}\right)-h\left(B_{s \wedge \tau_{V}}\right)\right)^{2}\right]=E^{x}\left[\int_{s \wedge \tau_{V}}^{t \wedge \tau_{V}}\left|\nabla h\left(B_{u}\right)\right|^{2} d u\right] \rightarrow 0, \quad s, t \uparrow \tau_{V} .
$$

So $h\left(B_{t \wedge \tau_{V}}\right)$ converges in $L^{2}\left(P^{x}\right)$ as $t \uparrow \tau_{V}$. By (2.17), (2.23) and the martingale convergence theorem this limit is necessarily equal to $h^{x}$.

Thus we have proved that (2.9)-(2.13) hold with $h^{x}$ instead of $h^{*}$. We proceed to show that $h^{x}$ does not depend on $x$ (modulo sets of measure 0 ):

Choose arbitrary $y \in V$. By adding an extra finely open set $U_{y}$ if necessary we may assume that the sequence $\left\{V_{n}\right\}$ constructed above contains $y$ (as well as $x$ ) for large enough $n$. Let $\mathscr{B}_{n}$ denote the $\sigma$-algebra generated by $\left\{B_{\tau_{k}} ; k \geqslant n\right\}$. Then if $n$ is large enough

$$
P^{y}\left|\mathscr{B}_{n} \ll P^{x}\right| \mathscr{B}_{n} .
$$

Therefore by (2.17)

$$
h_{n}(\omega) \rightarrow h^{x}(\omega) \text { a.s. } P^{y}
$$


On the other hand, by the same argument as above applied to $P^{y}$ there exists a function $h^{y} \in L^{2}\left(P^{y}\right)$ such that

$$
h_{n} \rightarrow h^{y} \text { in } L^{2}\left(P^{y}\right)
$$

and

$$
h_{n}(\omega) \rightarrow h^{y}(\omega) \text { a.s. } P^{y} .
$$

Combining (2.25) and (2.27) we see that

$$
h^{x}(\omega)=h^{y}(\omega) \text { a.s. } P^{x} .
$$

So we put $h^{*}=h^{x}$, and Theorem 1 is proved.

REMARK. Theorem 1 raises the following question: When is $h^{*}$ a genuine boundary function? In other words, when is $h^{*} B_{\tau_{V}}$-measurable, i.e. of the form $g\left(B_{\tau_{V}}\right)$ for some function $g \in L^{2}\left(\partial V, \lambda_{x}\right)$ ? Any function of the form $g\left(B_{\tau_{V}}\right)$ is $\mathfrak{B}^{V}$-measurable (since $B_{\tau_{V}}=\lim _{t \uparrow \tau_{V}} B_{t}$ ), but in general the family of $\mathfrak{B}^{V}$-measurable functions may also contain functions which are not of this type. For example, if

$$
V=\left\{\left(x_{1}, x_{2}\right) ; x_{1}^{2}+x_{2}^{2}<1\right\} \backslash\left\{\left(x_{1}, 0\right) ; x_{1}<0\right\} \subset \mathbf{R}^{2}
$$

and

$$
h\left(x_{1}, x_{2}\right)=\operatorname{Arg}\left(x_{1}+i x_{2}\right)=\operatorname{Im}\left(\log \left(x_{1}+i x_{2}\right)\right), \quad\left(x_{1}, x_{2}\right) \in V,
$$

then $h$ has different boundary values as the $B_{t}$ approach a point $\left(x_{1}, x_{2}\right)$ on the negative real axis from above or below. So $h^{*}$ is not $B_{\tau_{\nu}}$-measurable in this case.

Using Theorem 1 we obtain the following characterizations of finely harmonic functions satisfying (1.2).

THEOREM 2. Let $h$ be a finely harmonic function on a fine domain $V \subset \mathbf{R}^{d}$ with $a$ Green function $G$. Then the following are equivalent:

(i) $\int_{V}|\nabla h(y)|^{2} G(x, y) d m(y)<\infty$ for all $x \in V$.

(ii) There exists a $\mathfrak{B}^{V}$-measurable function $h^{*} \in L^{2}\left(P^{x}\right)$ for all $x$ such that

$$
h(x)=E^{x}\left[h^{*}\right] \text { for all } x \in V .
$$

(iii) For all $x \in V$ there exists a number $M_{x}<\infty$ such that

$$
E^{x}\left[h^{2}\left(B_{\tau}\right)\right]<M_{x}
$$

for all stopping times $\tau<\tau_{V}$.

Proof. (i) $\Rightarrow$ (ii) by Theorem 1 .

(ii) $\Rightarrow$ (iii). Suppose (ii) holds. Choose $x \in V$. Let $\tau<\tau_{V}$ be a stopping time. First assume that $\tau<\tau_{n}$ for some $n$. Then since $h^{*}$ is $\mathfrak{B}^{V}$-measurable $\theta_{\tau} h^{*}=h^{*}$ (see e.g. (7.16) in [13]) and

$$
\begin{aligned}
E^{x}\left[h^{2}\left(B_{\tau}\right)\right] & =E^{x}\left[\left(E^{B_{\tau}}\left[h^{*}\right]\right)^{2}\right]=E^{x}\left[\left(E^{x}\left[\theta_{\tau} h^{*} \mid B_{\tau}\right]\right)^{2}\right] \\
& =E^{x}\left[\left(E^{x}\left[h^{*} \mid B_{\tau}\right]\right)^{2}\right] \leqslant E^{x}\left[\left(h^{*}\right)^{2}\right]=M_{x} .
\end{aligned}
$$

In the general case we apply the above argument to $\tau \wedge \tau_{n}$ and obtain $E^{x}\left[h^{2}\left(B_{\tau \wedge} \tau_{n}\right)\right]$ $\leqslant M_{x}$. Letting $n \rightarrow \infty$ we get (iii). 
(iii) $\Rightarrow$ (i). Let $x \in V$. Let $V_{n}=V_{n}^{x}$ and $\tau_{n}=\tau_{n}^{x}$ be as in Lemma 4. Then

$$
\begin{aligned}
M_{x} & \geqslant E^{x}\left[h^{2}\left(B_{\tau_{n}}\right)\right]=h^{2}(x)+\int_{V_{n}}|\nabla h(y)|^{2} G(x, y) d m(y) \\
& \rightarrow h^{2}(x)+\int_{V}|\nabla h(y)|^{2} G(x, y) d m(y) \text { as } n \rightarrow \infty,
\end{aligned}
$$

and (i) follows.

3. Removable singularities. We now apply the existence of stochastic boundary values to prove the polar sets are removable singularity sets for finely harmonic functions with finite Dirichlet integral wrt the Green measure.

Theorem 3. Let $U \subset \mathbf{R}^{d}$ be a fine domain with a Green function $G$ and let $h$ be $a$ finely harmonic function on $V=U \backslash F$, where $F$ is a polar set. Suppose

$$
\int_{U}|\nabla h(y)|^{2} G(x, y) d m(y)<\infty \quad \text { for all } x \in U .
$$

Then $h$ extends to a finely harmonic function in $U$.

Proof. By Theorem 1 there exists a function $h^{*} \in L^{2}\left(P^{z}\right)$ such that

(3.2) $\lim _{t \uparrow \tau_{V}} h\left(B_{t}\right)=h^{*}(\omega)=h(z)+\int_{0}^{\tau_{V}}(\nabla h)\left(B_{s}\right) d B_{s}$ a.s. $P^{z}$, for all $z \in V$.

Moreover,

$$
h(z)=E^{z}\left[h^{*}\right] \text { for all } z \in V .
$$

Since $F$ is polar we have $\tau_{V}=\tau_{U}$ a.s. $P^{z}$ and therefore

$$
\lim _{t \uparrow \tau_{U}} h\left(B_{t}\right)=h^{*}(\omega)=h(z)+\int_{0}^{\tau_{U}}(\nabla h)\left(B_{s}\right) d B_{s} \text { a.s. } P^{z}, \quad \text { for all } z \in V .
$$

Thus a natural candidate for the required harmonic extension of $h$ would be $\tilde{h}(x)=E^{x}\left[h^{*}\right], x \in U$. However, it is not clear that $h^{*} \in L^{1}\left(P^{x}\right)$ if $x \in F$. In fact, it turns out that $h^{*} \in L^{2}\left(P^{x}\right)$ for all $x \in F$, as the following argument shows.

Fix $x \in F$. By the quasi-Lindelöf principle we can find an increasing sequence $\left\{U_{n}\right\}$ of finely open sets such that $\bar{U}_{n} \subset U, x \in \bigcup_{n=1}^{\infty} U_{n}$ and $K=U \backslash \bigcup_{n=1}^{\infty} U_{n}$ is polar.

Now apply Theorem 1 to the restriction $h \mid U_{n} \backslash F$ of $h$ to the set $U_{n} \backslash F$. This gives that there exists a function $h^{*, n} \in L^{2}\left(P^{z}\right)$ such that

$$
\lim _{t \uparrow \sigma_{n}} h\left(B_{t}\right)=h^{*, n}(\omega)=h(z)+\int_{0}^{\sigma_{n}}(\nabla h)\left(B_{s}\right) d B_{s} \text { a.s. } P^{z}
$$

for all $z \in U_{n} \backslash F$, where $\sigma_{n}=\tau_{U_{n} \backslash F}$. Since $h$ is finely continuous on $U \backslash F$ we have by Lemma 2 that

$$
\lim _{t \uparrow \sigma_{n}} h\left(B_{t}\right)=h\left(B_{\sigma_{n}}\right) \text { a.s. } P^{z}, \quad z \in U_{n} \backslash F .
$$

Since $F$ is polar we have

$$
\sigma_{n}=\tau_{n} \text { a.s. } P^{z}, \quad z \in U_{n} \backslash F, \text { where } \tau_{n}=\tau_{U_{n}} .
$$


Therefore, by combining (3.5)-(3.7) we obtain

$$
h\left(B_{\tau}\right)=h(z)+\int_{0}^{\tau_{n}}(\nabla h)\left(B_{s}\right) d B_{s} \text { a.s. } P^{z}, \quad z \in U_{n} \backslash F .
$$

Hence, for $n>m$

$$
h\left(B_{\tau_{n}}\right)-h\left(B_{\tau_{m}}\right)=\int_{\tau_{m}}^{\tau_{n}}(\nabla h)\left(B_{s}\right) d B_{s} \text { a.s. } P^{z}, \quad z \in U_{m} \backslash F .
$$

Since $P^{x}\left|\mathfrak{B}_{m} \ll P^{z}\right| \mathfrak{B}_{m}$ if $m$ is large enough, we conclude that

$$
h\left(B_{\tau_{n}}\right)-h\left(B_{\tau_{m}}\right)=\int_{\tau_{m}}^{\tau_{n}}(\nabla h)\left(B_{s}\right) d B_{s} \text { a.s. } P^{x} .
$$

Now assume that (3.1) holds. This gives as before that $h_{n}=h\left(B_{\tau_{n}}\right)$ converges in $L^{2}\left(P^{x}\right)$ and pointwise a.e. $P^{x}$ to a function $g^{x} \in L^{2}\left(P^{x}\right)$. But we also know that $h_{n} \rightarrow h^{*}$ a.e. $P^{z}$ for $z \in \bigcup_{n=1}^{\infty} U_{n} \backslash F$ and therefore $h_{n} \rightarrow h^{*}$ a.e. $P^{x}$. We conclude that

$$
g^{x}=h^{*} \in L^{2}\left(P^{x}\right)
$$

So we define

$$
\tilde{h}(x)=E^{x}\left[h^{*}\right], \quad x \in U
$$

Then $\tilde{h}=h$ in $U \backslash F$.

We claim that $\tilde{h}$ is finely harmonic in $U$. To see this choose $x \in U$ and a fine neighbourhood $D$ of $x$ such that $\bar{D} \subset U$. Let $T$ be the first exit time from $D$. Since $K \cup F$ is polar we must have $T<\tau_{n}$ for some $n$. Hence since $h^{*}$ is $\mathfrak{B}^{V}$-measurable we get by the strong Markov property

$$
\tilde{h}(x)=E^{x}\left[h^{*}\right]=E^{x}\left[E^{x}\left[h^{*} \mid B_{T}\right]\right]=E^{x}\left[E^{B_{T}}\left[h^{*}\right]\right]=\int_{\partial D} \tilde{h}(z) d \lambda_{x}^{D}(x),
$$

so that $\tilde{h}$ satisfies the required mean value property.

As pointed out to me by B. Fuglede it is possible to give a stronger, pointwise version of Theorem 3 by combining Theorem 3 with Theorem 2.4 in [11], mentioned in the introduction:

THEOREM 4. Let $U$ be as in Theorem 3 and let $h$ be a finely harmonic function on $U \backslash F$, where $F$ is a polar set. Suppose

$$
\int_{U}|\nabla h(y)|^{2} G\left(x_{0}, y\right) d m(y)<\infty
$$

for some point $x_{0} \in F$. Then $h$ extends to a finely harmonic function in $U \backslash\left(F \backslash\left\{x_{0}\right\}\right)$.

COROllary 1. Let $U$ be as in Theorem 3 and let be a finely harmonic function in $U \backslash\left\{x_{0}\right\}$, where $x_{0}$ is some point in $U$. Suppose (3.13) holds. Then h extends to a finely harmonic function in $U$.

REMARKs. (1) Note that Theorem 3 also gives a proof of Theorem 9.15 in Fuglede [8]: If $h$ is bounded in $V$, then $h^{*}$ is bounded and so $\tilde{h}(x)=E^{x}\left[h^{*}\right]$ is the required harmonic extension of $h$. Using the identity (3.10) we also see that if $h$ is bounded in $V$ then (3.1) holds. 
(2) The results above also apply to the case where we have $m$-dimensional finely harmonic functions $h: V \subset \mathbf{R}^{n} \rightarrow \mathbf{R}^{m}$. In particular, they apply to the finely harmonic morphisms $\phi=\left(\phi_{1}, \ldots, \phi_{m}\right): V \rightarrow \mathbf{R}^{m}$. In that case the condition that

$$
\int_{V}\left|\nabla \phi_{j}\right|^{2}(y) G(x, y) d m(y)<\infty, \quad 1 \leqslant j \leqslant m, x \in V,
$$

can be seen to be satisfied if the exit time $\tau_{\phi(V)}$ for Brownian motion in $\mathbf{R}^{m}$ has a finite expected value:

$$
E^{z}\left[\tau_{\phi(V)}\right]<\infty \text { for all } z \in \phi(V)
$$

To see that (3.15) implies (3.14) we apply the fact that $\phi$ is a Brownian path preserving function (see (2.3), (2.4) and Theorem 1 in [14]). Since

$$
\phi\left(B_{\sigma_{t}-1}\right) \in \phi(V) \text { for all } t<\sigma_{\tau_{V}},
$$

where $\sigma_{t}=\int_{0}^{t}\left|\nabla \phi_{j}\right|^{2}\left(B_{s}\right) d s\left(\sigma_{t}\right.$ does not depend on $\left.j\right)$, we have

$$
\sigma_{\tau_{V}} \leqslant \tau_{\phi(V)}
$$

and therefore

$$
\begin{aligned}
\int_{V}\left|\nabla \phi_{j}\right|^{2}(y) G(x, y) d m(y) & =E^{x}\left[\int_{0}^{\tau_{V}}\left|\nabla \phi_{j}\right|^{2}\left(B_{s}\right) d s\right] \\
& =E^{x}\left[\sigma_{\tau_{V}}\right] \leqslant E^{\phi(x)}\left[\tau_{\phi(V)}\right]<\infty,
\end{aligned}
$$

as asserted. In view of (1.4) it is natural to regard the finely harmonic morphisms satisfying (3.14) as generalized $H^{2}$-functions. The implication $(3.15) \Rightarrow(3.14)$ then corresponds to the now-well-known connection between classical $H^{2}$-functions and exit times from their image sets. See Burkholder [2].

(3) Consider the special case of an ordinary harmonic function $h$ on a domain (in the ordinary topology) $V$ in $\mathbf{R}^{d}$. Then the conclusions of Theorems 1,2 and 3 hold in particular if we replace the condition (1.2) by (1.1), since-as noted in the introduction-(1.1) implies (1.2) in that case.

In this case the assumption (3.1) of Theorem 3 can be relaxed to (3.17) below.

CoROllaRY 2. Let $U$ be a Greenian domain in $\mathbf{R}^{d}$ (in the ordinary topology), $F a$ relatively closed polar subset of $U$ and $h$ a harmonic function on $U \backslash F$. Suppose

$$
\int_{U}|\nabla h(y)|^{2} G(x, y) d m(y)<\infty \quad \text { for all } x \in U \backslash F .
$$

Then $h$ extends to a finely harmonic function in $U$.

Proof. From Theorem 1 we obtain $h^{*}=\lim _{t \uparrow \tau_{U}} h\left(B_{t}\right) \in L^{2}\left(P^{z}\right)$ for $z \in U \backslash F$ as before. By the Harnack inequalities we have $h^{*} \in L^{2}\left(P^{x}\right)$ for $x \in F$ also, so

$$
\tilde{h}(x)=E^{x}\left[h^{*}\right], \quad x \in U,
$$

is the desired harmonic extension. 
Finally, we note that by combining Corollary 2 with remark (2) we obtain

COROLlaRY 3. Let $U$ be a Greenian domain in $\mathbf{C}^{n}, F$ a relatively closed polar subset of $U$ and $\phi$ a (complex) analytic function on $U \backslash F$. Suppose that

$$
E^{z}\left[\tau_{\phi(U \backslash F)}\right]<\infty \quad \text { for all } z \in \phi(U \backslash F) .
$$

Then $\phi$ extends to an analytic function on $U$.

ACKNOWLEDGements. I am very grateful to B. Fuglede, T. Kolsrud, T. Lyons and the referee for many valuable comments.

\section{REFERENCES}

1. M. Brelot, On topologies and boundaries in potential theory, Lacture Notes in Math., vol. 175, Springer-Verlag, 1971.

2. D. L. Burkholder, Exit times of Brownian motion, harmonic majorization, and Hardy spaces, Adv. in Math. 26 (1977), 182-205.

3. K. L. Chung, Lectures from Markov processes to Brownian motion, Springer-Verlag, 1982.

4. A. Debiard and B. Gaveau, Differentiabilité des functions finement harmoniques, Invent. Math. 29 (1975), 111-123.

5. J. L. Doob, Application to analysis of a topological definition of smallness of a set, Bull. Amer. Math. Soc. 72 (1966), 579-600.

6. B_ Boundary properties of functions with finite Dirichlet integrals, Ann. Inst. Fourier (Grenoble) 12 (1962), 573-621.

7. E. B. Dynkin, Markov processes. I, Springer-Verlag, 1965.

8. B. Fuglede, Finely harmonic functions, Lecture Notes in Math., vol. 289, Springer-Verlag, 1970.

9. $\quad$ Functions harmoniques et functions finement harmoniques, Ann. Inst. Fourier (Grenoble) 24 (1974), 77-91.

10. __ Functions BLD et functions finement surharmonique, Université de Paris 6: Séminaire de Théorie du Potentiel, No. 6, 1982, pp. 126-157.

11. __ Integral representation of fine potentials, Math. Ann. 262 (1983), 191-214.

12. M. Fukushima, Dirichlet forms and Markov processes, North-Holland, Kodansha, 1980.

13. B. Øksendal, An introduction to stochastic differential equations with applications, Springer-Verlag (to appear).

14. Finely harmonic morphisms, Brownian path preserving functions and conformal martingales, Invent. Math. 75 (1984), 179-187.

Mathematical Institute, University of Oslo, OSLo 3, NoRWay

Current address: Department of Mathematics, University of California, Los Angeles, California 90024 